\title{
Relación entre el autoconcepto social, el clima familiar y el clima escolar con el bullying en estudiantes de secundaria
}

\author{
Relationship between Social Self-Concept, Family Climate and School Climate \\ with Bullying in Secondary Students \\ Relação entre 0 autoconceito social, 0 clima familiar e 0 clima escolar \\ com 0 bullying em estudantes do ensino secundário
}

\author{
Ángel Alberto Valdés Cuervo* \\ Instituto Tecnológico de Sonora \\ Ernesto Alonso Carlos Martínez ${ }^{* *}$ \\ Instituto Tecnológico Superior de Cajeme
}

Doi: dx.doi.org/10.12804/apl32.03.2014.07

\section{Resumen}

Este estudio determinó la relación de las variables autoconcepto social, clima familiar y clima escolar con la presencia de bullying en estudiantes de secundarias públicas de un estado del noroeste de México. De un total de 930 estudiantes encuestados se seleccionaron para participar en el estudio a 195 (20.1\%), los cuales reportaron un promedio de tres o más conductas agresivas hacia los pares durante el último mes. Utilizándose una regresión lineal múltiple se encontró que las variables Clima Escolar (Beta estandarizado $=-.491)$ y Clima Familiar (Beta estandarizado $=-.407$ ), explican una parte importante de la violencia entre estudiantes $\left(R^{2}=.74\right)$. El hallazgo de que las variables escolares y familiares se relacionan de manera significativa con el bullying evidenció la utilidad del enfoque ecológico en el análisis de esta problemática.

Palabras clave: Bullying; estudiantes; factores de riesgo; familia; escuela. fibstract

The study determined the relationship between the variables social self, family climate and school climate with the presence of bullying in public high school students of a northwestern state of Mexico. From a total of 930 students who were surveyed 195 were selected to participate in the study (20.1\%), who reported an average of three or more aggressive behaviors toward peers during the last month. Using a multiple linear regression it was determined that the variables School Climate (standardized Beta $=-.491$ ) and Family Climate (standardized Beta $=-.407)$, explain a significant portion of violence among students $\left(R^{2}=.74\right)$. The fact that school and family variables were significantly related to bullying revealed the usefulness of the ecological approach in the analysis of the problem.

Keywords: Bullying; students; risk factors; family; school.

\footnotetext{
* Ángel Alberto Valdés Cuervo, Departamento de Educación, Instituto Tecnológico de Sonora.

** Ernesto Alonso Carlos Martínez, Instituto Tecnológico Superior de Cajeme.

La correspondencia relacionada con este artículo debe ser enviada Ángel Alberto Valdés Cuervo, a la Calle 5 de Febrero 818 Sur CP. 85000 , Ciudad Obregón, Sonora. Tel: (644) 410 0927. Correo electrónico: angel.valdes@itson.edu.mx.
}

Para citar este artículo: Valdés, A., \& Martínez, E. A. (2014). Relación entre el autoconcepto social, el clima familiar y el clima escolar con el bullying en estudiantes de secundaria. Avances en Psicología Latinoamericana, 32(3), 447-457. doi: dx.doi.org/10.12804/ ap132.03.2014.07 


\section{Resumo}

O estudo determinou a relação das variáveis autoconceito social, clima familiar e clima escolar com a presença de bullying em estudantes do ensino secundário público de um estado do noroeste do México. De um total de 930 estudantes inquiridos se selecionaram para participar no estudo a 195 (20,1\%), os quais reportaram uma média de três ou mais condutas agressivas com os pais durante o último mês. Utilizando uma regressão linear múltipla encontrou-se que as variáveis Clima Escolar (Beta estandardizado = -.491) e Clima Familiar (Beta estandardizado $=-.407$ ), explicam uma parte importante da violência entre estudantes $(\mathrm{R} 2=.74)$. $\mathrm{O}$ achado de que as variáveis escolares e familiares se relacionam de forma significativa com o bullying, evidenciou a utilidade do enfoque ecológico na análise desta problemática. Palavras-chave: Bullying; estudantes; fatores de risco; família; escola.

En México, al igual que en muchos otros países, el bullying es un fenómeno al que se le ha comenzado a brindar una mayor atención en las escuelas de los diversos niveles educativos, esto se debe, en gran parte, al aumento del número de investigaciones acerca de esta temática. Resultan de particular interés, para el presente trabajo, los estudios que se enfocan en el nivel de educación básica, pues evidencian que el bullying en sus distintas manifestaciones (físico, social y psicológico) es un fenómeno que se presenta con frecuencia en las escuelas mexicanas de este nivel (Castillo \& Pacheco, 2008; Haro, García \& Reidl, 2013; Instituto Nacional de Evaluación Educativa [INEE], 2006).

Stephenson y Smith (2008) definen el bullying como una situación en la que un individuo o grupo de estudiantes acosan intencionalmente a un compañero o grupo de compañeros de escuela. Este fenómeno se caracteriza por (a) existencia de una clara diferencia de poder entre el agresor y la víctima, (b) intención expresa de infligir dolor físico y/o emocional, (c) carácter repetitivo y (d) naturaleza relacional (Goossens, Olthof \& Delkker, 2006; Olweus, 1993).

El bullying influye de manera negativa en la función que debe ejercer la escuela, relativa a la formación de ciudadanos capaces de convivir en sociedades democráticas (Elliot, 2008; Ortega \& Del Rey, 2008). Las víctimas de los actos violentos en dicha etapa pueden presentar efectos negativos como el rechazo a asistir a clases, disminución del desempeño académico, ansiedad, depresión, retraimiento, baja autoestima e incluso suicidio (O’Brennan, Bradshaw \& Sawyer, 2009, Olweus, 1993). Por su parte, los agresores presentan con frecuencia, tanto en la juventud como en la vida adulta, conductas antisociales, abuso de alcohol, problemas para asumir responsabilidades, desempleo, rupturas matrimoniales y trastornos psiquiátricos (Coloroso, 2004; Olweus, 1993; Pearce, 2008).

Las causas del bullying son multifactoriales por lo que resulta conveniente abordarlas desde un enfoque ecológico que integre diversas perspectivas teóricas (Coloroso, 2004; Ortega \& Córdova, 2010; Stephenson \& Smith, 2008). Lo anterior se debe a que la violencia entre pares es resultado de las interacciones de los estudiantes con sus diversos contextos de desarrollo, por lo que es conveniente considerar niveles de análisis individuales, grupales, sociales y culturales en la explicación de este (López, Bilbao \& Rodríguez, 2011; Postigo, González, Montoya \& Ordoñez, 2013; Swearer, Espegale \& Napolitano, 2009). Asumiendo el valor del enfoque ecológico en la comprensión del bullying, en el presente estudio se decidió considerar la relación de este fenómeno con variables ubicadas en los niveles del individuo, la familia y la escuela.

Se partió de la teoría desarrollista para el análisis de los factores individuales asociados con la violencia entre pares, en particular del modelo que sostiene que el bullying se origina por un mecanismo de desconexión moral en el individuo, lo que le dificulta sentir pena o culpa por el daño ocasionado a los otros y que incluso permite que valore de forma positiva estos actos agresivos (Ahmed \& Braithwaite, 2004; Morrison, 2006; Sánchez, Ortega \& Menesini, 2012). En específico, se seleccionó, dentro de las posibles variables que se han estudiado desde esta teoría, el autoconcepto social que se forma mediante la percepción del individuo con respecto a sus habilidades en los contextos en los que mantiene relaciones sociales (Goñi \& Fernández, 2007). Aunque los estudios aún no resultan 
concluyentes, un grupo importante de ellos sostiene que los agresores presentan un autoconcepto social más alto que las víctimas e incluso que los propios observadores (Estévez, Martínez, Moreno \& Musitu, 2006; Torre, Cruz, Villa \& Casanova, 2008), lo cual hace suponer que valoran como positivas sus conductas agresivas.

La revisión de los factores del contexto familiar relacionados con la presencia del bullying se realizó desde la teoría del aprendizaje social propuesta por Bandura (1973). En este marco, se considera al clima familiar como una variable relevante para la explicación del bullying ya que integra aspectos referentes a las relaciones y al funcionamiento familiar (Moos, Moos \& Trickett, 1984). Al respecto, diversos estudios señalan que el clima familiar de los estudiantes con conductas de bullying se caracteriza por la presencia de una alta conflictividad y expresiones de violencia (Cava, Musitu \& Murgui, 2006; Christie-Mizell, 2003; Estévez, Murgui, Moreno \& Musitu, 2007; Pontzer, 2010; Valdés, Carlos \& Torres, 2012). Estos aspectos se asocian con dificultades en la comunicación, escaso afecto y apoyo por parte de los padres, naturalización de la violencia como forma de relación y deficiente regulación de la conducta de los hijos por la familia (Bowes, Maughan, Caspi, Moffit \& Arseneault, 2010; Corvo \& DeLara, 2010; Espegale \& Swearer, 2009; Estévez, et ál., 2006; Idsoe, Solli \& Cosmovici, 2008).

El contexto escolar se abordó desde la teoría de la conducta restaurativa, donde se sostiene que el bullying es el resultado de comportamientos que procuran construir una identidad dentro de un ambiente percibido como violento (Postigo, et ál., 2013). Dentro del conjunto de variables abordadas en el marco de esta teoría, resalta el clima social escolar, el cual es construido por la calidad de las relaciones entre los agentes educativos (Loukas \& Murphy, 2007). Al respecto, se ha encontrado que cuando se favorece el respeto y la convivencia, a la vez que se le brinda seguridad a los estudiantes, existe una menor frecuencia de comportamientos violentos entre los pares (Collie, Shapka \& Perry, 2011; Good, McIntosch \& Gietz, 2011; Greene, 2006; Johnson, 2009; Stephenson \& Smith, 2008).
Aunque en la literatura académica internacional existen abundantes estudios que abordan la relación de las variables consideradas en el presente estudio con el bullying, por lo general estas investigaciones las han abordado de manera aislada, evidenciándose con ello la ausencia de un enfoque ecológico en el análisis de la problemática y una falta de integración entre las diversas teorías explicativas del bullying. Estas dos características de los estudios anteriores limitan la posibilidad de brindar una comprensión más holística del fenómeno de la violencia escolar (Swearer, et ál., 2009).

Por otra parte, en México predominan los estudios descriptivos en el abordaje de la problemática de la violencia escolar. En este contexto, el presente estudio constituye un aporte original al tema en el sentido en que, desde un enfoque ecológico, se procuró la integración de diversas teorías, así como se pretendió identificar variables asociadas con la violencia escolar en una población de estudiantes de nivel medio básico de un estado del noroeste de la república mexicana.

Desde el punto de vista teórico, se pretende mostrar la utilidad del enfoque ecológico y de la integración de diversas perspectivas teóricas, como herramientas metodológicas válidas para abordar el bullying. Además, se espera contribuir a ampliar la comprensión acerca del bullying en estudiantes mexicanos de educación básica, ya que se indaga acerca de variables situadas en diferentes contextos de desarrollo de los estudiantes para comprender la violencia entre pares en las escuelas mexicanas.

Desde el punto de vista práctico, el estudio, al identificar variables relevantes asociadas con la violencia escolar en el contexto de escuelas de nivel medio, puede ser útil al orientar las acciones preventivas que se planean por los diversos actores educativos. Lo anterior es de vital importancia en el diseño e implementación de los programas de prevención e intervención de la violencia escolar, los cuales deben concentrar sus estrategias en la atención de aquellos aspectos que mayor incidencia tienen en los diversos casos de violencia escolar (Olweus, 1994).

Atendiendo a lo anterior, el presente estudio responde a lo siguiente: ¿en qué medida de ma- 
nera conjunta las variables 'Autoconcepto Social', 'Clima Familiar' (medido por los factores de 'Apoyo-Tolerancia', 'Cultural-Recreativo' y 'Desarrollo Personal') y el 'Clima Escolar' (evaluado en las dimensiones relativas al 'Centro Escolar' y al 'Profesor') explican la frecuencia de conductas de bullying en estudiantes de secundaria?

Se partió de la hipótesis de que las variables 'Autoconcepto Social', 'Clima familiar' y el 'Clima escolar' constituyen un modelo que permite explicar una parte significativa de la varianza de los reportes de bullying por parte de los estudiantes.

\section{Método}

\section{Participantes}

La población estuvo compuesta por estudiantes de secundarias públicas de tres municipios del sur del estado de Sonora. Por medio de un muestreo aleatorio simple, que tomó como base una probabilidad de éxito del $50 \%$ y un nivel de confianza del 95\% ( $p=.50 ; q=.5)$, se seleccionaron 930 estudiantes. A partir de esta muestra inicial, y de manera intencional, se seleccionaron a los estudiantes que participaban en situaciones de bullying en el rol de agresores. Como punto de corte para la identificación de estos, se asumió el propuesto por Solberg y Olweus (2003), quienes consideraron que tres o más informes de actos de violencia reportados hacia compañeros durante el último mes permitían considerar a un estudiante como agresor.

Atendiendo al criterio antes mencionado, se identificaron 195 (20.1\%) estudiantes que reportaron un promedio de tres o más conductas agresivas hacia los pares durante el último mes. Estos tuvieron una edad promedio de 13.8 años, predominó entre ellos el sexo masculino (67.1\%). De los estudiantes identificados con conductas agresivas hacia los pares, $34.6 \%$ cursaban el primer año, $25.7 \%$ el segundo y $29.7 \%$ el tercer año de secundaria.

\section{Instrumentos}

Caracterización de la violencia escolar. Con base en los instrumentos elaborados por Del Rey y Ortega (2005) y Raviv et ál. (2001) se diseñó para el estudio un cuestionario que midió la frecuencia con la que los estudiantes reportaron llevar a cabo conductas agresivas hacia los pares durante el último mes. El instrumento evaluó, por medio de nueve ítems, diversos tipos de agresiones: (a) físicas (tres ítems), tales como 'Le has pegado a tus compañeros'; (b) sociales (tres ítems), por ejemplo 'Evitas que algunos compañeros se integren a los juegos' y (c) psicológicas (tres ítems), como 'Amenazas a tus compañeros'.

Las respuestas utilizaron una escala tipo Likert que osciló desde 'Nunca', 'Casi Nunca' (una o dos veces al mes), 'A veces' (tres a cuatro veces al mes), 'Casi siempre' (cinco a siete veces al mes) y 'Siempre' (más de siete veces al mes). El instrumento mostró una confiabilidad de .90 medida por medio del Alfa de Cronbach.

Autoconcepto social. En este trabajo se utilizó la adaptación elaborada por Yáñez, Valdés y Vera (2012) del cuestionario de De la Rosa y Díaz (1986). Con el propósito de fortalecer las propiedades psicométricas del instrumento, se realizó un análisis factorial con el método de Máxima Verosimilitud y rotación Oblimin, donde se confirmó la estructura propuesta por los autores de dos factores denominados 'Autoconcepto prosocial' y 'Autoconcepto Disocial'. En este análisis se alcanzó a explicar el $45.8 \%$ de la varianza del constructo.

En las instrucciones se le pidió al individuo que identificara el grado en que poseía ciertas características psicológicas. Se contestó utilizándose una escala tipo Likert con siete opciones de respuesta que osciló desde 'Nada' (1) hasta 'Totalmente' (7). Los puntajes se codificaron de manera tal que cuando fueran más altos indicaran un mejor autoconcepto social.

La confiabilidad de los puntajes de la escala en el presente estudio fue de .88 , lo que permitió considerar como buena la consistencia interna del instrumento.

Clima familiar. Se utilizó el instrumento desarrollado por Carlos, Valdés y Reyes (en prensa) para medir el clima familiar, que se basó en el desarrollado por Moos et ál., (1984). Este nuevo instrumento se realizó atendiendo a las inconsistencias encontradas 
en la estructura factorial de la escala antes mencionada (Boake \& Salmon, 1983; Canto, Esquivel \& Valdés, 2005; Oliver, May \& Handal, 1988).

Carlos, et ál., (en prensa) reportaron haber obtenido, por medio de un análisis factorial confirmatorio, con la misma muestra del presente estudio, un modelo sustentable de evaluación del clima familiar $\left(X^{2}=47.4 ; p=.039 ; G F I=.99 ; R S M R=.09\right)$ que explicó el $72 \%$ de la varianza total de los puntajes. Este se integró por 10 ítems agrupados en tres factores: 'Apoyo-Tolerancia', el cual evaluó el grado de cooperación y ayuda entre los miembros mediante cuatro ítems, por ejemplo 'Cuando existe desacuerdo en mi familia se procura mantener la calma'; el otro factor 'Cultural-Recreativo', midió la existencia de prácticas que promueven el sano esparcimiento y la estimulación intelectual con tres ítems, un ejemplo es 'Mi familia asiste a eventos culturales' y 'Desarrollo Personal', que abordó la importancia que le otorga la familia al desarrollo de habilidades en sus integrantes para la vida independiente mediante tres ítems, por ejemplo 'Mi familia le da importancia a ser exitosos en lo que hacemos'.

El instrumento se contestó mediante una escala tipo Likert con cinco opciones de respuesta que osciló desde 'Nunca' (1), 'Casi Nunca' (2), 'A Veces' (3), 'Casi siempre' (4) y 'Siempre' (5), siendo indicativos los mayores puntajes de un mejor clima familiar.

En el presente estudio se determinó la confiabilidad de los puntajes mediante el Alfa de Cronbach obteniéndose un valor de .87 , que lo ubica con una alta consistencia interna.

Clima Social Escolar. Se utilizó el cuestionario desarrollado por Trianes, Blanca, De La Morena, Infante y Raya (2006), quienes refirieron que al utilizar un análisis factorial extrajeron dos factores que explicaron el $93 \%$ de la varianza total de los puntajes: (a) 'Referente al Centro Escolar' que midió las relaciones del estudiante con la escuela en general con ocho reactivos, por ejemplo 'Mi escuela es un lugar seguro' y (b) 'Referente al Profesor', el cual consideró la naturaleza de las relaciones de los estudiantes con los profesores e involucró a seis reactivos, tales como 'Me siento cómodo hablando con mis profesores de mis problemas'.
Este instrumento se respondió con una escala tipo Likert con cuatro opciones de respuesta que van desde 'Totalmente en Desacuerdo' (1), 'En desacuerdo' (2), 'De acuerdo' (3) y 'Totalmente de acuerdo' (4), donde los puntajes mayores indicaron un clima escolar más favorable.

En el presente estudio los resultados de este instrumento obtuvieron un Alfa de Cronbach igual a .83 , lo cual indica una buena confiabilidad aceptable en su medición.

\section{Procedimiento para la recolección de información y análisis de datos}

En la obtención de la información se cuidó el cumplir con los criterios éticos establecidos en la investigación científica. Para lo anterior, se solicitó la autorización a los directores de las escuelas y el consentimiento por escrito a los padres de los estudiantes. Se invitó a los estudiantes a participar de manera voluntaria en la aplicación del instrumento, garantizándoseles la confidencialidad de los resultados y se entregó un resumen a las autoridades de las escuelas y los representantes de las sociedades de padres de familias.

Para el análisis de los datos se utilizó el SPSS. v. 17 y estadísticos multivariados de interdependencia, en particular una regresión lineal múltiple con el método paso a paso.

\section{Resultados}

El análisis de los datos se realizó mediante una regresión lineal múltiple y, como paso previo a la obtención del modelo, se valoró el cumplimiento de los supuestos implícitos en un análisis de este tipo: (a) Linealidad, se confirmó por medio del análisis de los gráficos de regresión parcial y de los residuos; (b) Normalidad, la cual se valoró por medio del histograma de residuos; (c) Independencia, lo que se evidenció con los resultados de la prueba Durbin-Watson obteniéndose un valor de 2.12, lo cual denotó ausencia de correlación entre los residuos; (d) Homocedasticidad, se realizó un gráfico de los valores predichos tipificados frente a los residuos estandarizados y se comprobó que la varianza de los residuos se comportó de manera 
uniforme por medio de los distintos niveles de la variable dependiente y (e) Ausencia de colinealidad, se demostró con la presencia de una tolerancia próxima a 1 .

Antes de abordar directamente los resultados de la regresión, cabe mencionar que se constató la existencia de correlaciones significativas entre cada una de las variables independientes con respecto a la variable dependiente (promedio de reportes de bullying en el último mes). Se apreció que todas las variables independientes presentaron correlaciones estadísticamente significativas con la variable dependiente (ver tabla 1).

Tabla1

Correlaciones entre las variables consideradas

en el modelo

$\begin{array}{ccc}\text { Clima } & \text { Clima } & \text { Autoconcepto } \\ \text { escolar } & \text { familiar } & \text { social }\end{array}$

Promedio de reportes

de conductas agresi-

vas hacia los pares en

$-.446^{*}-.391 * \quad .230 *$

el último mes

${ }^{*} p \leq .05$

La variable dependiente (reportes bullying) se relacionó de manera negativa con las variables independientes 'Clima Escolar' y 'Clima Familiar', lo cual indica que climas familiares y escolares favorables se asocian con una menor presencia de conductas de bullying (ver tabla 1). Para el caso del 'Autoconcepto Social', se encontró una relación positiva, lo cual sugiere una mayor frecuencia de reportes de bullying en los estudiantes que se percibían socialmente de manera más positiva.

Se constató la hipótesis de que la pendiente de la recta de regresión es distinta a cero y por lo tanto las variables están linealmente relacionadas (ver tabla 2).

Tabla 2

Resultados del modelo de regresión

\begin{tabular}{lcrcc}
\hline Modelo & Suma de cuadrados & gl & $F$ & $p$ \\
\hline Regresión & 138.32 & 2 & 348.8 & .000 \\
Residual & 48.00 & 193 & & \\
Total & 186.32 & 195 & & \\
\hline$p \leq .05$ & & & &
\end{tabular}

Los resultados evidenciaron que la frecuencia de bullying se relacionó de manera lineal con el Clima social escolar (Beta estandarizado $=-.491$ ) y Clima familiar (Beta estandarizado $=-.407)$, los que conjuntamente explican el $74 \%$ de la varianza de la variable dependiente, ya que se obtuvo un $R^{2}$ de .74. La variable autoconcepto social fue excluida del modelo por no resultar significativa dentro de este (ver tabla 3 ).

Tabla 3

Variables que explican la frecuencia de bullying en estudiantes de secundaria

\begin{tabular}{lcccc}
\hline & Constante & $\begin{array}{c}\text { Beta } \\
\text { Estandarizado }\end{array}$ & $t$ & $p$ \\
\hline Constante & 8.286 & & 36.14 & .000 \\
$\begin{array}{l}\text { Clima social } \\
\text { escolar }\end{array}$ & & -.491 & -8.168 & .000 \\
$\begin{array}{l}\text { Clima } \\
\text { familiar }\end{array}$ & & & & \\
\hline
\end{tabular}

Nota: $R^{2}=.74$; Durbin-Watson= $2.12 ; F=348.84 ; p=.05$

\section{Discusión}

A partir del propósito del estudio, que fue determinar las relaciones del autoconcepto social, el clima familiar y el clima escolar con la presencia de conductas violentas entre estudiantes en las escuelas secundarias, es conveniente señalar que los resultados evidenciaron que dos de estas variables (clima familiar y clima escolar) forman un modelo que logra explicar una parte significativa de la varianza de los reportes de bullying por parte de los estudiantes.

Al ubicarse las variables consideradas en dos de los sistemas estudiados - escolar y familiar-el estudio evidenció la necesidad de que la problemática de la violencia escolar sea abordada desde una perspectiva ecológica, donde deben considerarse intervenciones en los diferentes niveles en que se desarrolla el estudiante (Morrison, 2006; Ortega \& Del Rey, 2008; Swearer et ál., 2009; Tutty, 2008). Además, se mostró la utilidad de integrar diversas perspectivas teóricas en el análisis de las variables relacionadas con el bullying escolar, aunque esto puede parecer evidente a simple vista, por lo general, 
ha sido obviado en los diversos estudios que abordan el problema desde una u otra posición teórica.

El hecho de que el clima escolar manifieste relación con la presencia de bullying entre estudiantes, es consistente con lo reportado en estudios previos (Bayar \& Ucanok, 2012; Cava, Musitu, Buelga y Murgui, 2010; Quintana, Montgomery, Malaver \& Ruíz, 2012; Yoneyama \& Rigby, 2006). Lo anterior, permite afirmar que el bullying se asocia con la presencia de un ambiente escolar negativo que se caracteriza entre otros aspectos por la percepción del estudiante de inseguridad, poco apoyo social en la escuela y dificultades para comunicarse abiertamente con los profesores y directivos (Ahmed \& Braithwaite, 2006; Nesdale \& Pickering, 2006; Ortega \& Del Rey, 2008). Estos resultados sostienen la importancia de que se generen en las escuelas estrategias para la creación de un ambiente escolar que facilite la prevención del bullying. Es de mencionar que la acción de las instituciones educativas en los programas anti-bullying se ve favorecida por la posibilidad que poseen las escuelas para coordinar las acciones de los diferentes actores involucrados en la problemática, como los estudiantes, docentes, padres de familia y la comunidad en general (Epstein, 2011).

Los hallazgos del presente estudio también confirman los reportes de otras investigaciones, en el sentido de que un clima familiar negativo se asocia con la presencia de conductas violentas en la escuela, y por lo tanto deben ser objeto de acciones en los programas de prevención de este fenómeno (Besag, 2008; Estévez, et ál., 2006; Olweus, 1993; Swearer, et ál., 2009). Desde esta perspectiva, las intervenciones dirigidas a las familias con problemas de violencia deben centrarse en el desarrollo de normas, valores y relaciones entre los integrantes del núcleo familiar que contribuyan en el respeto, la confianza y la consideración de las necesidades de cada uno de los miembros de la familia. Un buen clima familiar permite a los padres ofrecer a los hijos modelos funcionales de relaciones sociales que después puedan utilizar en otros contextos de desarrollo (Espegale \& Swearer, 2009).

Si bien, el autoconcepto social no resultó una variable significativamente relacionada con la frecuencia de reportes de bullying dentro del modelo de regresión, es necesario recordar que de forma independiente mostró una relación significativa positiva, aunque de baja intensidad, con la frecuencia de reportes de bullying. Este hallazgo coincide con lo reportado en otros estudios que refieren que los agresores se perciben de manera positiva desde el punto de vista social (Johnson \& Lewis, 1999; Kaukianen, et ál., 2002; Torres, et ál., 2008), y es consistente con la hipótesis de que el agresor es reforzado socialmente por los pares (Caravita, Di Blasio \& Salmivalli, 2009; Salmivalli \& Nieminen, 2002). Este reforzamiento de la agresión le otorga un carácter instrumental asociado con la obtención de prestigio y popularidad social que es necesario tener en cuenta en el diseño de los programas preventivos en el tema.

Los resultados del estudio permitieron concluir que la violencia que se presenta entre pares en la escuela debe ser abordada desde una perspectiva ecológica, ya que solo al tener en cuenta los diversos contextos de desarrollo de los alumnos es que esta problemática puede ser comprendida y abordada de manera efectiva. Por otra parte, se sugiere que los programas de prevención y gestión de la convivencia escolar en este nivel consideren incluir acciones dirigidas a intervenir en las variables que en el presente estudio se identificaron como asociadas con la violencia escolar.

El hecho de que los programas preventivos tengan en cuenta los resultados de la investigación empírica contextualizada a nuestra realidad, sin lugar a dudas puede contribuir a mejorar su efectividad y favorecer que las escuelas puedan cumplir a cabalidad su objetivo definitivo: formar individuos que sean capaces de convivir y participar de manera armónica, dentro de una sociedad democrática, ya que cualquier forma de violencia es contraria al logro de este trascendental fin.

El estudio tiene como una de sus limitaciones el haber considerado una sola variable en cada uno de los contextos de desarrollo. Esto implicó no integrar otros aspectos que pueden ser relevantes dentro de cada nivel de análisis, y, por ende, la imposibilidad de generar modelos de análisis multinivel de la problemática del bullying. Por otra parte, el tipo de análisis estadístico utilizado no permite visualizar posibles interacciones entre las variables 
independientes, lo cual indiscutiblemente limita la comprensión del fenómeno.

Lo anterior indica que es necesario ampliar este estudio con la inclusión de variables ubicadas en los distintos contextos, y generar modelos multiniveles $\mathrm{y}$ estructurales que permitan comprender el grado en que cada uno de ellos coadyuva a la comprensión de la violencia entre pares en el contexto escolar.

\section{Referencias}

Ahmed, E., \& Braithwaite, V. (2004). "What, me ashamed?" Shame management and school bullying. Journal of Research in Crime and Delinquency, 41(3), 269-294. doi: 10.1177/0022427804266547

Ahmed, E., \& Braithwaite, V. (2006). Forgiveness, reconciliation, and shame: Three key variables in reducing school bullying. Journal of Social Issues, 62(2), 347-370. doi: 10.1111/j.15404560.2006.00454.x

Bandura, A. (1973). Aggression: A social learning theory analysis. New York: Prentice-Hall.

Bayar, Y., \& Ucanok, Z. (2012). School social climate and generalized peer perception in traditional and cyberbullying status. Educational Sciences: Theory \& Practices, 12(4), 2352-2358. Recuperado de www.edam.com.tr/kuyeb/en/

Besag, V. (2008). Padres y maestros trabajando juntos. En M. Elliot (Ed.), Intimidación. Una guía práctica para combatir el miedo en las escuelas (pp. 219-238). México Distrito Federal: Fondo de Cultura Económica.

Boake, C., \& Salmon, P. (1983). Demographic correlates and factor structure of the family environment scale. Journal of Clinical Psychology, 39(1), 95-100. doi: 10.1002/1097-4779(198301)39:1>3.0.CO;2-3

Bowes, L., Maughan, B., Caspi, A., Moffit, T., \& Arseneault, L. (2010). Families promote emotional and behavioural resilience to bullying: evidence of on environmental effect. The Journal of Child Psychology and Psychiatry, 51(7), 809-817. doi:10.1111/j.1469-7610.2010.02216.x

Canto, J., Esquivel, L., \& Valdés, A. (2005). Perfil del estudiante de nuevo ingreso a las licenciaturas de la
Universidad Autónoma de Yucatán. (Reporte Técnico). Mérida: Universidad Autónoma de Yucatán.

Caravita, S., Di Blasio, P., \& Salmivalli, C. (2009). Unique and interactive effects of empathy and social status on involvement in bully. Social Development, 18(1), 140-163. doi: 10.1111/j.14679507.2008.00465.x

Carlos, E., Valdés, A., \& Reyes, C. (en prensa). Desarrollo de un modelo empíricamente sustentable para medir el clima familiar. En R. Díaz, S. Rivera, \& I. Reyes (Eds.), La Psicología Social en México. Mérida: AMEPSO.

Castillo, C., \& Pacheco, M. (2008). Perfil del maltrato (bullying) entre estudiantes de secundaria en la ciudad de Mérida, Yucatán. Revista Mexicana de Investigación Educativa, 13(38), 825-842.

Cava, M., Musitu, G., Buelga, S., \& Murgui, S. (2010). The relationships of family and classroom environment with peer relational victimization: An analysis of their gender differences. The Spanish Journal of Psychology, 13(1), 156-165.

Cava, M., Musitu, G., \& Murgui, S. (2006). Familia y violencia escolar: el rol mediador de la autoestima y la actitud hacia la autoridad institucional. Psicothema, 18(003), 367-373.

Christie-Mizell, C. (2003). Bullying: the consequences of interparental discord and child's selfconcept. Family Process, 42(2), 237-251. doi: 10.1111/j.1545-5300.2003.42204.x

Collie, R., Shapka, J., \& Perry, N. (2011). Predicting teacher commitment: the impact of school climate and social-emotional learning. Psychology in the Schools, 48(10), 1034-1048. doi: 10.1002/ pits. 20611

Coloroso, B. (2004). The bully, the bullied and the bystander. New York: Harper Colling.

Corvo, K., \& DeLara, E. (2010). Towards an integrated theory of relational violence: Is bullying a risk factor for domestic violence? Aggression and Violent Behavior, 15(3), 181-190. doi: 10.1016/j. avb.2009.12.001

Del Rey, R., \& Ortega, R. (2005). Violencia interpersonal y gestión de la disciplina, Revista Mexicana de Investigación Educativa, 10(26), 805-832. 
Elliot, M. (2008). Intimidadores y víctimas. En M. Elliot (Ed.), Intimidación. Una guía práctica para combatir el miedo en las escuelas (pp. 39-55). México, Distrito Federal: Fondo de Cultura Económica.

Epstein, J. (2011). School, Family and Community Partnerships (2 ${ }^{\mathrm{da}}$ ed.). New York: Westview Press.

Espegale, D., \& Swearer, S. (2009). Contributions of three social theories to understanding bullying perpetration and victimization among school-aged youth. En M. Harris (Ed.), Bullying, rejection \& peer victimization. A Social Cognitive Neuroscience perspective (pp. 151-170). New York: Springer.

Estévez, E., Martínez, B., Moreno, D., \& Musitu, G. (2006). Relaciones familiares, rechazo entre iguales y violencia escolar. Cultura y Educación, 18(34), 335-344.

Estévez, E., Murgui, S., Moreno, D., \& Musitu, G. (2007). Estilos de comunicación familiar, actitud hacia la autoridad institucional y conducta violenta del adolescente en la escuela. Psichotema, 19(1), 108-113.

Good, C., McIntosh, K., \& Gietz, C. (2011). Integrating bullying prevention into schoolwide positive behavior support. Teaching Exceptional Children, 44(1), 48-56. Recuperado de http://cec.metapress. com/content $/ 5 \mathrm{gw} 3868 \mathrm{~m} 0668353 \mathrm{p} /$

Goossens, F., Olthof, T., \& Dekker, P. (2006). New participant role scales: Comparison between various criteria for assigning roles and indications for their validity. Aggressive Behavior, 32(4), 343-357. doi: 10.1002/ab.20133

Goñi, E., \& Fernández, A. (2007). Los dominios social y personal del autoconcepto. Revista de Psicodidáctica, 12(2), 179-194.

Greene, M. (2006). Bullying in schools: A plea for measure of Human Rights. Journal of Social Issues, 62(1), 63-79. doi:10.1111/j.1540-4560.2006.00439

Idsoe, T., Solli, E., \& Cosmovici, M. (2008). Social psychological processes in family and school: more evidence on their relative etiological significance for bullying behavior. Aggressive Behavior, 34(5), 460-474. doi:10.1002/ab.20259

Haro, I., García, B., \& Reidl, L. (2013). Experiencia de culpa y vergüenza en situaciones de maltrato entre iguales en secundarias. Revista Mexicana de Investigación Educativa, 18(59), 1047-1075.

Instituto Nacional de Evaluación Educativa (2006). Informe sobre calidad de la Educación Básica. Violencia y disciplina en escuelas primarias y secundarias. México, Distrito Federal: INEE.

Johnson, S. (2009). Improving the school environment to reduce school violence: A review of the literature. Journal School Health, 79, 451-465. doi: 11010.1111/j.1746-1561.2009.00435.x

Johnson, D., \& Lewis, G. (1999). Do you like what to see? Self-perceptions of adolescent bullies. British Educational Research Association, 25(5), $665-$ 677. doi: 10.1080/0141192990250507

Kaukianen, A., Salmivalli, C., Lagerspetz, K., Tamminen, M., Vauras, M., Maki (2002). Learning difficulties, social intelligence, and self concept: Connections to bully-victim problems. Scandinavian Journal of Psychology, 43(3), 269-278. doi:10.1111/1467-9450.00295

López, V., Bilbao, M., \& Rodríguez, J. (2011). La sala de clases sí importa: incidencia del clima de aula sobre la percepción de intimidación y victimización entre escolares. Universitas Psychologica, 11(1), 91-101.

Loukas, A., \& Murphy, J. (2007). Middle school perceptions of school of climate: Examining protective functions on subsequent adjustment problems. Journal of School Psychology, 45(3), 293-309. doi:10.1016/j.jsp.2006.10.001.

Moos, R., Moos, B., \& Trickett, E. (1984). FES, WES, CIES, CES. Escalas de Clima Social. Madrid: TEA.

Morrison, B. (2006). School Bullying and Restorative Justice: Toward a Theoretical Understanding of the Role of Respect, Pride, and Shame. Journal of Social Issues, 62(2), 371-392. doi:1011/ j.1540.2006.00455.x

Nesdale, D., \& Pickering, K. (2006). Teacher's reaction to children's aggression. Social Development, 15(1), 109-127. doi:10.1111/j.14679507.2006.00332.x

O’Brennan, L., Bradshaw, C., \& Sawyer, A. (2009). Examining developmental differences in the socialemotional problems among frequent bullies, vic- 
tims, and bully/victims. Psychology in the Schools, 46 (2), 100-115. doi: 10.1002/pits.20357

Oliver, J., May, M. \& Handal, P. (1988). The factor structure of the family environment scale: factors derived from scales. Journal of Clinical Psychology, 44, 5, 723-727. doi: 10.1002/1097-4679

Olweus, D. (1993). Bullying at school. What we know and what we can do. Oxford: Blackwell Publishing.

Olweus, D. (1994). Annotation: Bullying at school. Basic facts and effects of a school based intervention program. Journal of Child Psychology Psychiatry, 35(7), 1171-1190.

Ortega, R., \& Córdova, F. (2010). Construir la convivencia para prevenir la violencia: Un modelo ecológico. En R. Ortega (Ed.), Agresividad injustificada, bullying y violencia escolar (pp. 299-320). Madrid: Alianza Editorial.

Ortega, R., \& Del Rey, R. (2008). La violencia escolar. Estrategias de prevención (4ta ed.). Madrid: Grao.

Pearce, J. (2008). ¿Qué se puede hacer con el agresor? En M. Elliot (Ed.), Intimidación. Una guía práctica para combatir el miedo en las escuelas (pp. 157-186). México, Distrito Federal: Fondo de Cultura Económica.

Pontzer, D. (2010). A theoretical test of bullying Behavior: Parenting, personality, and the bullying/ victim relationships. Journal of Family Violence, 25(3), 259-273. doi: 10.1007/s10896-009-9289-5

Postigo, S., González, R., Montoya, I., \& Ordoñez, A. (2013). Theoretical proposals in bullying research: a review. Anales de Psicología, 29(2), 413-425. doi: 10.6018/analesps.29.2.148251

Quintana, A., Montgomery, J., Malaver, C., \& Ruíz, G. (2012). Percepción del bullying, gestión de conflictos y clima escolar en directivos y docentes de centros educativos. Revista de Investigación en Psicología, 15(2), 35-47.

Raviv, A., Erel, O., Fox, N., Leavitt, L., Raviv, A., \& Dar, I. (2001). Individual measurement of exposure to everyday violence among schoolchildren across various setting. Journal of Community Psychology, 29(2), 117-140. doi: 10.1002/1520-6629

Salmivalli, C., \& Nieminen, E. (2002). Proactive and reactive aggression among school bullies, victims and bully-victims. Aggressive Behavior, 28, 30-44. doi: 10.1002/ab.90004

Sánchez, V., Ortega, R., \& Menesini, E. (2012). La competencia emocional de agresores y víctimas de bullying. Anales de Psicología, 28(1), 71-82. doi: 10.6018/analesps.28.1.140542

Solberg, M., \& Olweus, D. (2003). Prevalence estimation of school bullying with the Olweus bully/ victim questionnaire. Aggressive Behavior, 29(3), 239-268. doi: 10.1002/ab.10047

Stephenson, P., \& Smith, D. (2008). ¿Por qué algunas escuelas no tienen acosadores? En M. Elliot (Ed.), Intimidación. Una guía práctica para combatir el miedo en las escuelas (pp. 56-78). México, Distrito Federal: Fondo de Cultura Económica.

Swearer, S., Espegale, D., \& Napolitano, S. (2009). Bullying. Prevention \& Intervention. Realistic Strategies for Schools. New York: The Guilford Press.

Tamar, F. (2005). Maltrato entre escolares (bullying): estrategias de manejo que implementan los profesores al interior del establecimiento escolar. Psykhe, 14(1), 211-225.

Torre, J., Cruz, M., Villa, M., \& Casanova, P. (2008). Relaciones entre violencia escolar y autoconcepto multidimensional en adolescentes de Educación Secundaria Obligatoria. European Journal of Education and Psychology, 1(2), 57-70.

Trianes, M., Blanca, M., De La Morena, L., Infante, L., \& Raya, S. (2006). Un cuestionario para evaluar el clima social del Centro Escolar. Psicothema, 18(002), 272-277.

Tutty, L. (2008). Best practices in school-based bullying prevention programs: What works? In D. Pepler, \& W. Craig (Eds.), Understanding and addressing bullying: An International Perspective (pp. 144163). Kingston: Authothouse.

Valdés, A., Carlos, E., \& Torres, G. (2012). Diferencias en la situación socioeconómica, clima y ajuste familiar de estudiantes con reportes de bullying y $\sin$ ellos. Psicología desde el Caribe, 29(3), 616-631.

Yáñez, A., Valdés, A., \& Vera, J. (2012). Caracterización del autoconcepto de estudiantes sobresalientes. En R. Díaz-Loving, S. Rivera, \& I. Reyes (Eds.), 
Relación entre el autoconcepto social, el clima familiar y el clima escolar con el bullying en estudiantes de secundaria

Aportaciones actuales de la psicología social (pp. 169-173). Monterrey: AMEPSO.

Yoneyama, S., \& Rigby, K. (2006). Bully/victim student and classroom climate. Youth Studies Australia,
25(3), 34-41. Recuperado de www.acys.info/ysa/ issues/v.25_n2.3_2006/pp34_41_rigby.pdf 
